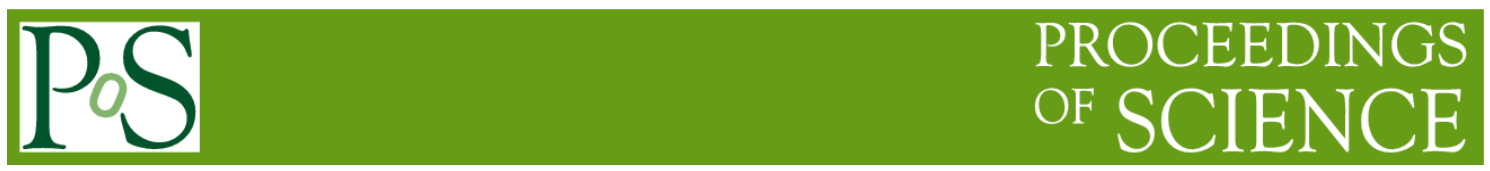

\title{
TOROIDAL MODELS OF THE FORCE-FREE MAGNETIC FIELD
}

\section{Anastasia Petukhova ${ }^{1}$}

Yu.G. Shafer Institute of Cosmophysical Research and Aeronomy, SB RAS, Yakutsk, Russia 31 Lenin Ave., Yakutsk, Russia

E-mail: petukhovaeikfia.ysn.ru

\section{Ivan Petukhov}

Yu.G. Shafer Institute of Cosmophysical Research and Aeronomy, SB RAS, Yakutsk, Russia 31 Lenin Ave., Yakutsk, Russia

E-mail: i_vaneikfia.ysn.ru

\section{Stanislav Petukhov}

Yu.G. Shafer Institute of Cosmophysical Research and Aeronomy, SB RAS, Yakutsk, Russia 31 Lenin Ave., Yakutsk, Russia

E-mail: petukhoveikfia.ysn.ru

The properties of four toroidal models of the magnetic flux rope are shown. The results of the analysis can be used to interpret in-situ observations of the magnetic flux rope and study Forbushdecrease in magnetic clouds.

\section{${ }^{1}$ Speaker}




\section{Introduction}

It is suggested that magnetic clouds are large-scale magnetic loops driven from the solar atmosphere to interplanetary space by coronal mass ejections (CMEs). For interpretation of insitu observations, the cylindrical model of the magnetic field called the Lundqvist solution is often used [1]. It is easy to see that the cylindrical model does not describe properties of field curvature. To interpret measurements, Marubashi [2] introduced a toroidal structure of the magnetic field. The parameters of magnetic clouds based on the cylindrical and toroidal models significantly differ in [3]: 1) orientation of the flux rope axis; 2) transverse radius (the flux rope radius) (radius in the toroidal model is less).

The cylindrical model is also applied to research Forbush decrease (FD) in a magnetic cloud [4]. However, the cylindrical model has its shortcomings as spatial distribution of cosmic rays (CR) depends on magnetic flux rope (MFR) due to their high mobility. Thus, the toroidal model is more appropriate for these studies $[5,6]$.

In this work we show and compare properties of four toroidal models for the force-free magnetic field, which can be used in various studies.

\section{Models of the force-free magnetic field}

It is generally suggested that the magnetic field in magnetic clouds is force-free. This field satisfies to the equation $\vec{\jmath} \times \vec{B}=0$, where $\vec{\jmath}$ is current density, $\vec{B}$ is magnetic field strenght. It follows that the current flows along the magnetic field $\vec{\jmath} \sim \vec{B}$. Taking into account Maxwell's equations, the equation system determing the force-free magnetic field can be written as

$$
\vec{\nabla} \times \vec{B}=\alpha \vec{B}, \quad \vec{\nabla} \vec{B}=0
$$

where $\alpha$ is a scalar. In the case when $\alpha$ is a constant or depends on coordinates, the field is called linear or nonlinear, respectively. The second equation from (1) takes into account the solenoidal condition.

\subsection{Miller \& Turner solution}

Miller and Turner calculated the magnetic field in a torus [7]. They used curvilinear coordinate system, its coordinates are connected with Cartesian coordinates by the following equations

$$
x=\left(R_{0}+\rho \cos \theta\right) \cos \varphi, \quad y=\left(R_{0}+\rho \cos \theta\right) \sin \varphi, \quad z=\rho \sin \theta,
$$

where $R_{0}$ is the torus axis radius, which locates in the X0Y plane; $\rho$ is the cross-section radius of the torus; $\theta$ is the angle in this plane counted from X0Y towards the Z-axis $(0 \leq \theta \leq$ $2 \pi) ; \varphi$ is the angle of this plane counted from the $\mathrm{X}$-axis towards the Y-axis $(0 \leq \varphi \leq 2 \pi)$. Taking into account the symmetry along the torus axis $(\partial / \partial \varphi=0)$, the solution of the system (1) can be presented as

$$
\begin{aligned}
& B_{\rho}=s_{1} s_{2} \frac{B_{0} \rho_{0}}{2 \alpha R_{0}} J_{0} \sin \theta, \quad B_{\theta}=-s_{1} s_{2} B_{0}\left[J_{1}-\frac{\rho_{0}}{4.8 R_{0}}\left(J_{0}+2.4 \frac{\rho J_{1}}{\rho_{0}}\right) \cos \theta\right], \\
& B_{\varphi}=s_{1} B_{0}\left(1-\frac{\rho \cos \theta}{2 R_{0}}\right) J_{0},
\end{aligned}
$$

where $J_{0}, J_{1}$ are the Bessel functions of the zero- and first- orders; $B_{0}$ is the magnetic field strength at the torus axis; $s_{1}= \pm 1, s_{2}= \pm 1$. Values of $s_{1}, s_{2}$ describe 4 types of MFR. Equations from (2) include the boundary conditions of $B_{\rho}\left(\rho_{0}\right)=0, B_{\varphi}\left(\rho_{0}\right)=0$ reached by the choice of an 
argument of the Bessel function as $\alpha= \pm 2.4 / \rho_{0}$, - the first root of the Bessel function of the zero-order. Solution (2) is approximate supposing that $\rho_{0} / R_{0} \ll 1$. Substituting (2) in (1), we obtain $\vec{\nabla} \times \vec{B}=\alpha \vec{B}+\vec{H}$, where $\vec{H}$ is discrepancy. The solenoidal condition is approximately fulfilled as well.

Figure a) shows the distributions of the relative magnetic field strength in the plane of the torus cross-section and the projections of the field lines located on the surface of toroids with different radii. Figure b) shows the projections of the field lines on the X0Y plane located on the surface of different toroids. Here we used toroidal surfaces with radii $\rho_{T}<\rho_{0}$ as toroids. As can be seen from the figure, the field lines are on the surface of each toroid. In this case, the field lines are represented as helices with a step depending on the toroid radius: the smaller the radius, the greater the step. The listed properties are characteristics of force-free magnetic fields. It can be seen from Figure a) that the axis of the magnetic field is slightly shifted from the center of the torus. The maximum value of the magnetic field strength is shifted to the center of the torus as well. The ratio between the magnetic field strengths at the torus axis and surface is limited by 2 . The calculation results shown in Figures a-h) are calculated with $R_{0} / \rho_{0}=5$.

\subsection{Modified Miller and Turner solution}

Romashets, Vandas [8] modified the Miller and Turner solution. They used the vector potential of the modified solution $\vec{A}^{(m)}=\frac{1}{\alpha} \vec{B}$, where $\vec{B}$ is the Miller and Turner solution (2). Thus, $\vec{B}^{(m)}=\vec{\nabla} \times \vec{A}^{(m)}=\frac{1}{\alpha} \vec{\nabla} \times \vec{B}=\frac{1}{\alpha}(\alpha \vec{B}+\vec{H})=\vec{B}+\frac{\vec{H}}{\alpha}$, where $\vec{B}^{(m)}$ is strength of the modified magnetic field, $\vec{H}$ is discrepancy of the Miller and Turner solution. Using (2), we obtain the components of the modified magnetic field

$$
\begin{aligned}
& B_{\rho}^{(m)}=\frac{B_{0} J_{0} \sin \theta}{2 \alpha R_{0}} \frac{\left(R_{0}-2 \rho \cos \theta\right)}{\left(R_{0}+\rho \cos \theta\right)}, \\
& B_{\theta}^{(m)}=\frac{B_{0}}{2 \alpha R_{0}\left(R_{0}+\rho \cos \theta\right)}\left[2 \alpha R_{0}^{2} J_{1}-R_{0} \cos \theta\left(J_{0}-\alpha \rho J_{1}\right)+\rho\left(2 J_{0}-\alpha \rho J_{1}\right) \cos ^{2} \theta\right], \\
& B_{\varphi}^{(m)}=B_{0} J_{0}\left(1-\rho \cos \theta / 2 R_{0}\right) .
\end{aligned}
$$

The modified solution is also approximate $\left(\rho_{0} / R_{0} \ll 1\right)$ and exactly satisfies the solenoidal condition.

Figures c, d) present the same as Figures a, b) for the modified Miller and Turner solution. The comparison of the figures shows that the Miller and Turner solution and the modified one are slightly different.

\subsection{Romashets and Vandas solution}

To calculate the magnetic field, the authors used the toroidal coordinate system [9], its parameters are related with the parameters of the Cartesian system as

$$
x=\frac{a \sinh \mu \cos \varphi}{\cosh \mu-\cos \eta}, \quad y=\frac{a \sinh \mu \sin \varphi}{\cosh \mu-\cos \eta}, \quad z=\frac{a \sin \eta}{\cosh \mu-\cos \eta} \text {. }
$$

Here $a$ is the coordinate system parameter, which is determined by size of a chosen torus $r_{0}=a / \sinh \mu_{0}, R_{0}=a \cosh \mu_{0} / \sinh \mu_{0}, R_{0} / r_{0}=\cosh \mu_{0}, a=\sqrt{R_{0}^{2}-r_{0}^{2}}=r_{0} \sqrt{\left(R_{0} / r_{0}\right)^{2}-1}$, where $r_{0}, R_{0}$ are the cross-section and torus axis radii, respectively. The parameters are determined by $\mu \geq \mu_{0}, 0 \leq \eta \leq 2 \pi, 0 \leq \varphi \leq 2 \pi$, where $\mu_{0}$ corresponds to surface of a chosen torus. 
The solution can be presented by the following equations

$B_{\mu}=0, B_{\eta}=-B_{0} \frac{\varepsilon \cosh \mu(\cosh \mu-\cos \eta)}{2 \sinh ^{3} \mu} F_{1}, B_{\varphi}=B_{0} \frac{\cosh \mu-\cos \eta}{\sinh \mu} F_{0}$,

where $F_{0}=F\left(\alpha_{0}, \beta_{0}, \gamma_{0}, \xi\right), \quad F_{1}=F\left(1+\alpha_{0}, 1+\beta_{0}, 1+\gamma_{0}, \xi\right)$ are hypergeometric functions, $\alpha_{0}=\left(1+\sqrt{1-4 \varepsilon^{2}}\right) / 4, \beta_{0}=\left(1-\sqrt{1-4 \varepsilon^{2}}\right) / 4, \gamma_{0}=1, \xi=-\sinh ^{2} \mu$.

All surfaces with $\mu=$ const are coordinate surfaces (toroids) in the toroidal coordinate system. The surface with $\mu=\mu_{0}$ coincides with the surface of a chosen torus and the surfaces with $\mu>\mu_{0}$ describe the toroids located inside a chosen torus. The surface with $\mu \rightarrow \infty$ degenerates into the torus axis. Solution (5) presents the magnetic field having two components whose field lines are located on the surface of the toroids. The hypergeometric function on the torus surface of $F\left(\alpha_{0}, \beta_{0}, \gamma_{0},-\sinh ^{-2} \mu_{0}\right)$ is used to determine values $\varepsilon$. As can be seen from (5), in this case $B_{\varphi}$ is zero on the torus surface. This condition is similar to that using the first root of the zero-order Bessel function in the Lundquist solution.

The ratios between the magnetic field components of the Cartesian and toroidal systems are obtained from

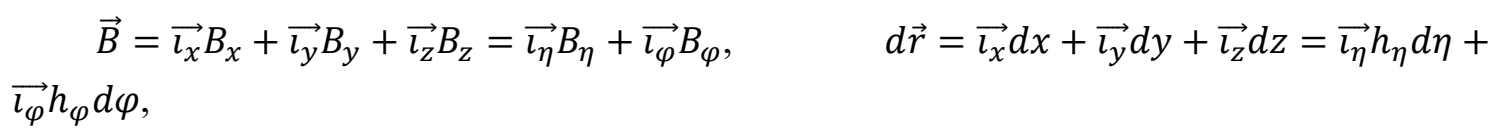

taking into account that the magnetic field in the toroidal system has 2 components $\left(B_{\mu}=\right.$ $0)$. Unit vectors of the coordinate systems are related as

$\overrightarrow{l_{\eta}}=\frac{1}{h_{\eta}}\left(\overrightarrow{l_{x}} \frac{\partial x}{\partial \eta}+\overrightarrow{l_{y}} \frac{\partial y}{\partial \eta}+\overrightarrow{l_{z}} \frac{\partial z}{\partial \eta}\right), \overrightarrow{l_{\varphi}}=\frac{1}{h_{\varphi}}\left(\overrightarrow{l_{x}} \frac{\partial x}{\partial \varphi}+\overrightarrow{l_{y}} \frac{\partial y}{\partial \varphi}\right)$

where $h_{\eta}, h_{\varphi}$ are the Lame coeffitients.

We calculate the partial derivatives using (4) and obtain

$B_{x}=B_{\eta} \sinh \mu \sin \eta \cos \varphi /(\cosh \mu-\cos \eta)-B_{\varphi} \sin \varphi$,

$B_{y}=B_{\eta} \sinh \mu \sin \eta \sin \varphi /(\cosh \mu-\cos \eta)+B_{\varphi} \cos \varphi$,

$B_{z}=B_{\eta}(\cosh \mu \cos \eta-1) /(\cosh \mu-\cos \eta)$.

Figures e, f) show the same as Figures a, b). As can be seen, the region with the maximum magnetic field strength is shifted toward the torus center. The ratio between the magnetic field strengths at the torus axis and surface is limited by 10 . The inhomogeneous distribution of the toroidal magnetic field increases with decreasing of $R_{0} / \rho_{0}$. Figure $\mathrm{g}, \mathrm{h}$ ) show the same as Figure a-f) with of $R_{0} / \rho_{0}=1.5$.

\subsection{Integral model}

Romashets and Vandas constructed the toroidal magnetic field using the Lundquist solution [10]. A generating torus has the cross-section radius of $\rho_{0}$ and the axis radius of $R_{0}$. The torus axis is located in the plane $\mathrm{X} 0 \mathrm{Y}$ of the laboratory Cartesian coordinate system and the torus center coincides with the system center. They add auxiliary cylinders with the cross-section radius of $\rho_{0}$, whose axes are located in the plane $\mathrm{X} 0 \mathrm{Y}$ and are the tangent to the radius circle of $R_{0}$, where $R_{0}$ is the torus axis. The magnetic field inside and outside the cylinder is the Lundquist solution.

The toroidal field is formed by the superposition of the magnetic fields of the auxiliary cylinders with the the angular size $\mathrm{d} \varphi$ :

$$
\begin{gathered}
B_{x}=-B_{0} \int_{0}^{2 \pi}\left(J_{1} z \cos \varphi / \rho-J_{0} \sin \varphi\right) d \varphi, B_{y}=-B_{0} \int_{0}^{2 \pi}\left(J_{1} z \sin \varphi / \rho-J_{0} \cos \varphi\right) d \varphi, B_{z}= \\
B_{0} \int_{0}^{2 \pi}\left(J_{1}\left(x \cos \varphi-y \sin \varphi-R_{0}\right) / \rho\right) d \varphi, \text { where } \rho=\sqrt{z^{2}+\left(x \cos \varphi-y \sin \varphi-R_{0}\right)^{2}}
\end{gathered}
$$


The Lundquist solution describes a force-free magnetic field with a constant value of $\alpha$, all the fields of the auxiliary cylinders are also force-free fields with the same value of $\alpha$, hence the superposition of the fields (6) is a force-free field with the same value $\alpha$.

The properties of the magnetic field are shown in Figures i, j). As can be seen, the magnetic field is located outside the torus, the projections of the field lines on the cross-section are significantly different from the circular ones. The ratio between the magnetic field strengths at the torus axis and surface is limited by 5 . Not all ratios allow to obtain MFR, field lines on the outer surface do not have a zero step.
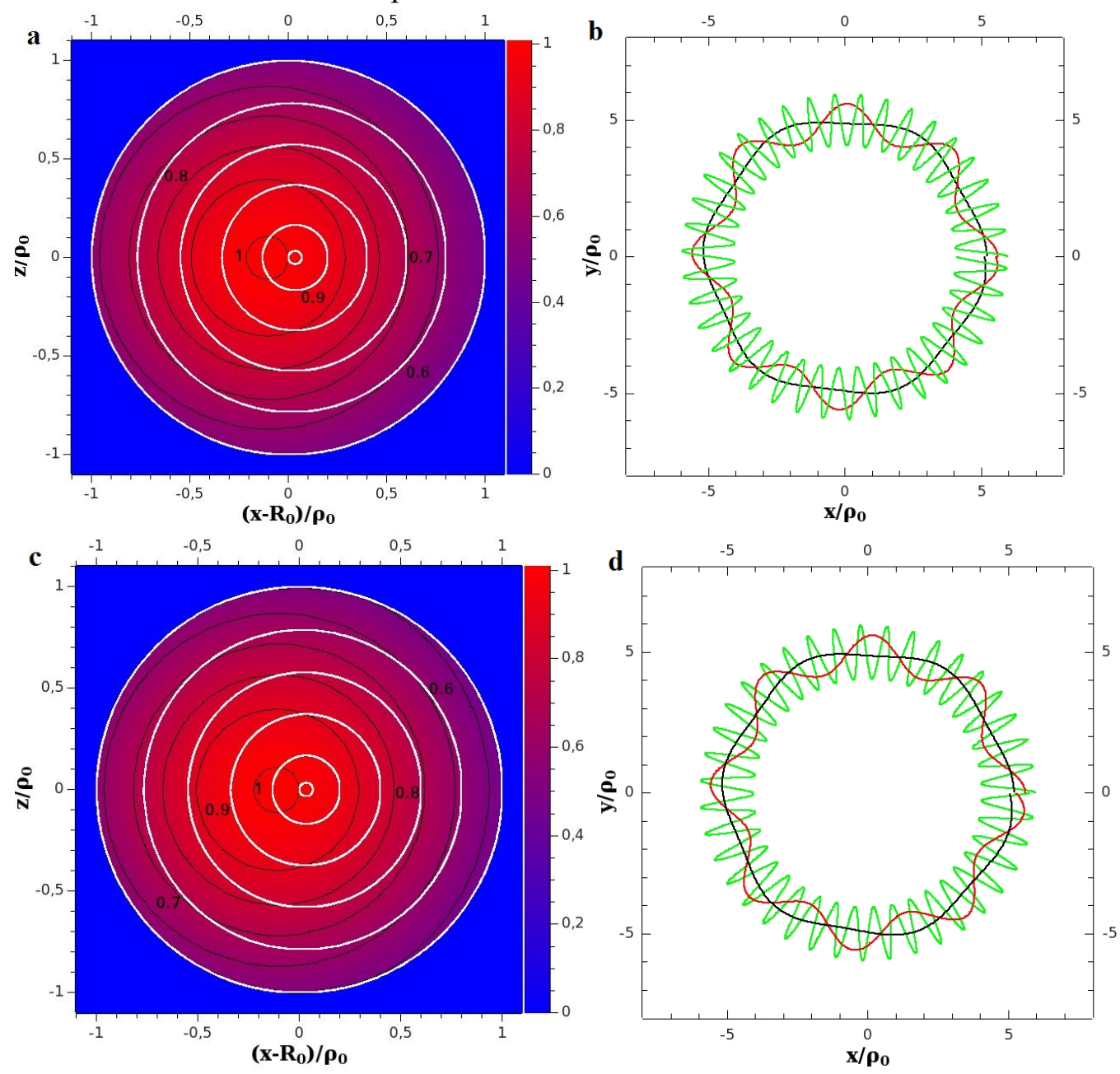

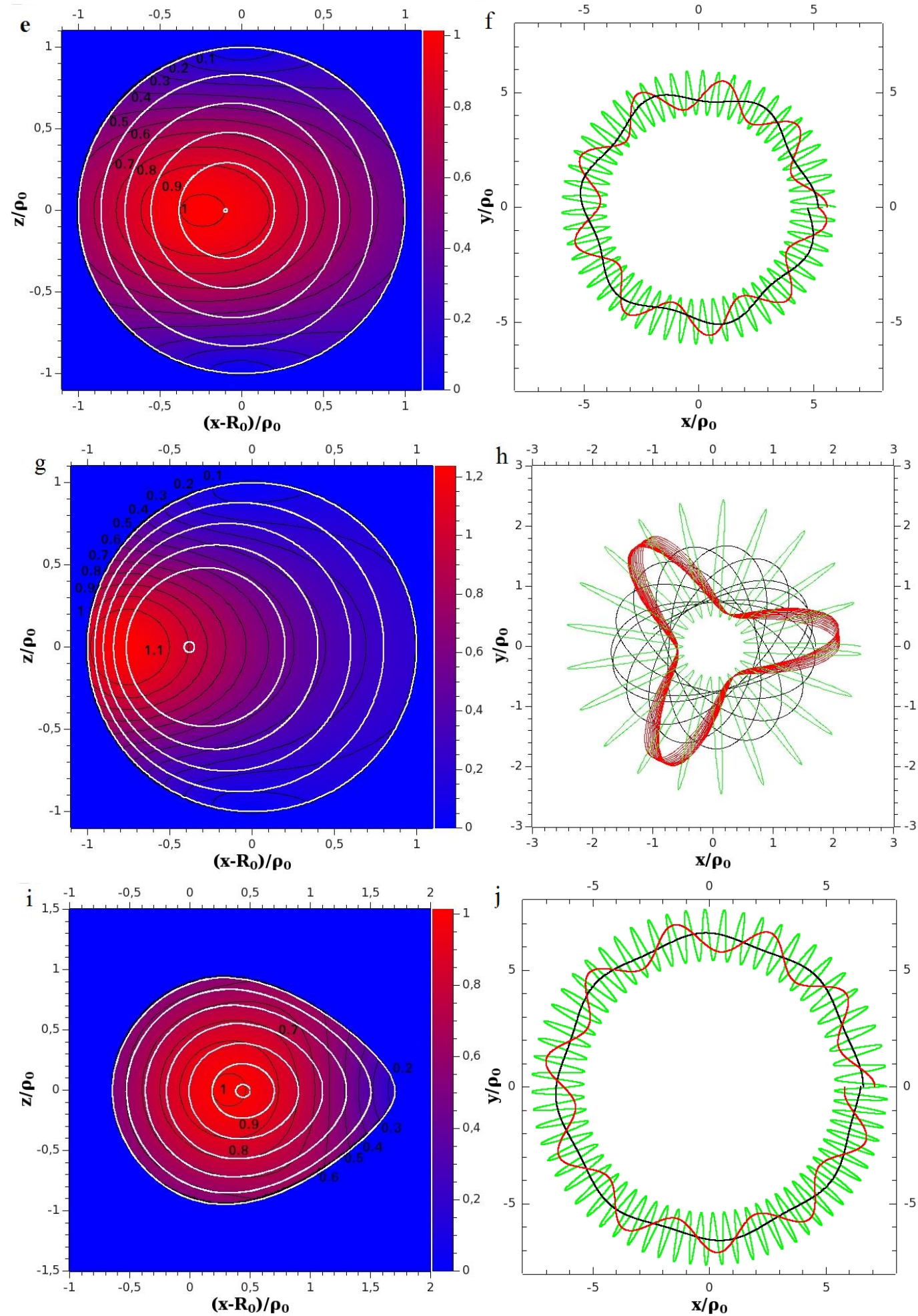

Figure: Magnetic field distribution in the cross-section plane and the projections of field lines on this plane located on the surface of toroids ( $a, c, e, g, i)$. The white curves denote radii of toroids with step $0.2 \rho_{0}$. The white circle is the torus axis. The black curves denote isolines, which bound regions with equal magnetic field magnitude. Projections of field lines located on the surface of toroids on X0Y plane $(b, d, f, h, j)$. The green, red, black curves correspond the toroids 
with radii $0.95 \rho_{0}, 0.6 \rho_{0}, 0.2 \rho_{0}$, respectively. The aspect ratio is $R_{0} / \rho_{0}=5$ (a-f), $R_{0} / \rho_{0}=1.5$ (g-h) and $R_{0} / \rho_{0}=6(\mathrm{i}-\mathrm{j})$.

\section{Conclusions}

The Miller \& Turner solution and the modified solution are slightly different. Due to approximation of the solutions, the ratio between the magnetic field strengths at the torus axis and surface is limited by 2. The magnetic field in the Romashets, Vandas solution is essentially asymmetric. The ratio is limited by 10 . The asymmetry and inhomogeneity of the magnetic field increase with decrease of the torus axis. For the integral solution, the ratio is limited by 5 and MFR it can be obtained not for any ratios.

The work is supported by Russian Foundation for Basic Research (Project №15-42-05085p_восток_a).

\section{References}

[1] L. F. Burlaga, Magnetic clouds and force-free fields with constant alpha, J. Geophys. Res. (1988) 93(A7): 7217-7224.

[2] K. Marubashi, Interplanetary Magnetic Flux Ropes and Solar Filaments in Coronal Mass Ejections Geophys. Monogr. Ser. (1997) 99: 147-156.

[3] K. Marubashi and R. P. Lepping Long-duration magnetic clouds: a comparison of analyses using torus- and cylinder-shaped flux rope models, Ann. Geophys. (2007) 25: 2453-2477.

[4] T. Kuwabara, J. W. Bieber, P. Evenson, et al., Determination of interplanetary coronal mass ejection geometry and orientation from ground-based observations of galactic cosmic rays, J. Geophys. Res. (2009) 114(A8): A05109.

[5] A.S. Petukhova, I.S. Petukhov \& S.I. Petukhov, Forbush decrease in the intensity of cosmic rays in a toroidal model of a magnetic cloud, Jetp Lett. (2015) 102: 697.

[6] A.S. Petukhova, I.S. Petukhov \& S.I. Petukhov, V. G. Grigoryev, Forbush decrease in the intensity of cosmic rays in a toroidal model of a magnetic cloud, Bull. Russ. Acad. Sci. Phys. (2017) 81: 532.

[7] G. Miller, L. Turner, Force free equilibria in toroidal geometry. Phys. Fluids. (1981) 24: 363-365.

[8] E. P. Romashets, M. Vandas, Interplanetary magnetic clouds of toroidal shapes, Proc. ISCS 2003 Symposium. (2003), 535-540

[9] E. P. Romashets, M. Vandas, Force-free field inside a toroidal magnetic cloud, Geophysical Research Letters (2003) 30: 2065-2069.

[10] E. P. Romashets, M. Vandas, Linear force-free field of a toroidal symmetry, Astronomy and Astrophysics (2009) 499: 17-20. 